\title{
ON H. S. RUSE'S THEOREMS CONCERNING PARALLEL FIELDS OF PLANES IN A RIEMANNIAN SPACE WITH INDEFINITE METRIC
}

\author{
YUNG-CHOW WONG
}

1. Introduction. In his paper [2], H. S. Ruse proved three theorems concerning parallel fields of partially-null planes in a Riemannian space with an indefinite metric. His proof was based on certain recurrence formulas obtained by applying A. G. Walker's recurrence formulas (cf. Walker [3] or Proposition (a) given below) to quasiorthogonal $n$-tuples of vectors constructed by the present author [4]. In this paper, we give a direct and simpler proof of Ruse's theorems and show that his theorems, which were established by him only locally, also hold globally.

Throughout this paper, the manifolds, fields of $r$-planes and so on are assumed to be of class $C^{\infty}$. We refer the reader to Walker [3], Ruse [2] and especially $\$ 1.1$ of Wong [5] for the definitions and terminology used and a proof of the following proposition which will be needed.

Proposition. Let $M$ be a connected, differentiable manifold with a linear connexion.

(a) A field II of $r$-planes on $M$ is parallel iff in each coordinate neighbourhood $U \subset M$ and for any local basis $\left\{X_{A}^{h}\right\}(1 \leqq A \leqq r)$ of $\Pi$ in $U$,

$$
\nabla_{k} X_{B}^{h}=L_{k B}^{A} X_{A}^{h} \quad(1 \leqq A, B \leqq r),
$$

where $\nabla$ denotes covariant differentiation and $L_{k B}^{A}$ are some covectors on $U$.

(b) Let $r$ be a fixed positive number. If for each point $u$ in $M$, there exists some coordinate neighbourhood $U \ni u$ in $M$, and a set of $r$ vectors $Y_{A}^{h}(1 \leqq A \leqq r)$ on $U$ such that

$$
\text { on } U: \nabla_{k} Y_{A}^{h}=L_{k A}^{B} Y_{B}^{h} \quad(1 \leqq A, B \leqq r),
$$

and

on $U \cap U^{*}: Y_{A^{*}}^{* h}={ }_{\phi_{A^{*}}^{A}}^{A} Y_{A}^{h}, \quad Y_{A}^{h}={ }_{\phi_{A}^{*}}^{A^{*}} Y_{A^{*}}^{* h} \quad\left(1 \leqq A, A^{*} \leqq r\right)$,

where $U^{*}$ is another coordinate neighbourhood and $\phi_{A^{*}}^{A^{*}}, \phi_{A}^{A^{*}}$ are functions on $U \cap U^{*}$, then the local fields of planes spanned by these sets of local vector fields piece together into a parallel field of planes on $M$.

Received by the editors August 25, 1966. 
2. The theorem. For a connected Riemannian manifold $M$ with an indefinite Riemannian metric, a vector subspace of any of its tangent spaces is said to be of nullity $s$ if one (and then every) of its mutually orthogonal basis contains exactly $s$ null vectors. As in Ruse [2], a parallel field of $r$-planes on $M$ each of which is of nullity $s$ will simply be called a parallel $r$-plane of nullity $s$. Ruse discovered that the existence on $M$ of a parallel $r$-plane of nullity $s$ for some special values of $r$ and $s$ possesses exceptional features in that it necessitates the existence of parallel planes other than its null part and its conjugate. We state his main results as follows.

Theorem. Let $M$ be a connected $n$-dimensional Riemannian manifold with an indefinite Riemannian metric.

(a) If $n=2 m$ and $M$ admits a null parallel $(m-1)$-plane, then it also admits two null parallel m-planes intersecting in the given $(m-1)$-plane. These planes are all contained in the parallel $(m+1)$-plane of nullity $m-1$ conjugate to the given $(m-1)$-plane.

(b) If $n=2 m$ and $M$ admits a parallel $m$-plane of nullity $m-1$, then (i) its null part $\Omega$ is a null parallel $(m-1)$-plane; (ii) it admits a pencil of parallel $m$-planes all intersecting in $\Omega$; of these, all except two are of nullity $m-1$, the exceptional two being wholly null $m$-planes; (iii) all the $m$-planes are contained in the parallel $(m+1)$-plane conjugate to $\Omega$, which is of nullity $m-1$.

(c) If $n=2 m+1$ and $M$ admits a parallel $m$-plane $\Pi$ of nullity $m-1$, then it also admits the following parallel planes: (i) the null $(m-1)$ plane $\Omega$, which is the null part of $\Pi$; (ii) two null m-planes $\Pi_{1}, \Pi_{2}$; (iii) the $(m+2)$-plane $\Omega^{\prime}$, of nullity $m-1$, conjugate to $\Omega$; (iv) the $(m+1)$-plane $\Pi^{\prime}$ of nullity $m-1$, conjugate to $\Pi$; $(\mathrm{v})$ the two $(m+1)$-planes $\Pi_{1}{ }^{\prime}, \Pi_{2}{ }^{\prime}$ both of nullity $m$, conjugate to $\Pi_{1}$ and $\Pi_{2}$. All these planes intersect in $\Omega$ and are contained in $\Omega^{\prime}$.

3. Proof of Theorem (a). We first observe that certain statements in the theorem are immediate consequences of the fact that the plane conjugate to any parallel plane is parallel, and the intersection and union of two parallel planes are both parallel planes (cf. Wong [5, Lemmas 1.6 and 1.7]; and Walker [3, Theorems 3.1 and 3.2]). Proof of these statements will be omitted.

We first prove that our assertions are true in any coordinate neighbourhood $U$ of $M$. Let $\left\{X_{A}\right\}, 1 \leqq A, B \leqq m-1$, be a normal basis of the given parallel null $(m-1)$-plane $\Pi$ on $U$. Then we have, by Proposition (a), that

$$
\nabla_{k} X_{B}^{h}=L_{k B}^{A} X_{A}^{h}
$$


Consider the system of $m-1$ equations in $W$ :

$$
X_{A} \cdot W=0,
$$

where $X_{\boldsymbol{A}} \cdot W$ denotes the inner product of the two vectors $X_{\boldsymbol{A}}$ and $W$ defined by the Riemannian metric on $M$. Since $\left\{X_{A}\right\}$ is a normal basis of a null $(m-1)$-plane, the vectors $X_{A}$ are independent and $X_{\boldsymbol{A}} \cdot X_{B}=0$. Therefore, equations (3.2) have exactly $2 m-(m-1)$ $=m+1$ independent solutions, and we may take them to be the $m+1$ vectors $X_{B}$ and two other vectors $Y_{1}$ and $Y_{2}$. Then $\left\{X_{A}, Y_{1}, Y_{2}\right\}$ form a basis of the $(m+1)$-plane $\Pi^{\prime}$ conjugate to $\Pi$. We now prove

Lemma. The vectors $Y_{1}, Y_{2}$ can be so chosen that

$$
Y_{1} \cdot Y_{1}=0, \quad Y_{2} \cdot Y_{2}=0, \quad Y_{1} \cdot Y_{2} \neq 0 .
$$

Proof. We first note that if at least one of $Y_{1} \cdot Y_{1}$ and $Y_{2} \cdot Y_{2}$ is zero, then $Y_{1} \cdot Y_{2} \neq 0$. For, if $Y_{1} \cdot Y_{1}=0$ and $Y_{1} \cdot Y_{2}=0$, say, then the system of $m+1$ independent equations in $W$ :

$$
X_{A} \cdot W=0, \quad Y_{1} \cdot W=0, \quad Y_{2} \cdot W=0
$$

would have $m$ independent solutions, namely, $X_{B}$ and $Y_{1}$, and this is impossible. We can now prove our lemma. If both $Y_{1} \cdot Y_{1}$ and $Y_{2} \cdot Y_{2}$ are zero, then our lemma is proved. If only one of them is zero, say, $Y_{1} \cdot Y_{1}=0$, we can replace $Y_{2}$ by $\tilde{Y}_{2}=\lambda Y_{1}+Y_{2}$, where $\lambda$ is the root of

$$
\tilde{Y}_{2} \cdot \tilde{Y}_{2}=2 \lambda Y_{1} \cdot Y_{2}+Y_{2} \cdot Y_{2}=0 \text {. }
$$

If neither $Y_{1} \cdot Y_{1}$ nor $Y_{2} \cdot Y_{2}$ is zero, we first replace $Y_{1}$ by $\tilde{Y}_{1}$ $=Y_{1}+\mu Y_{2}$, where $\mu$ (which may not be real) is a root of

$$
\tilde{Y}_{1} \cdot \tilde{Y}_{1}=Y_{1} \cdot Y_{1}+2 \mu Y_{1} \cdot Y_{2}+\mu^{2} Y_{2} \cdot Y_{2}=0,
$$

and then replace $Y_{2}$ by $\tilde{Y}_{2}=\tilde{\lambda} Y_{1}+Y_{2}$ for some suitable $\tilde{\lambda}$ as before. Hence our lemma is proved.

Let us now assume that $Y_{1}, Y_{2}$ have been chosen as in the above lemma. Then, $\left\{X_{A}, Y_{1}\right\}$ and $\left\{X_{A}, Y_{2}\right\}$ are normal bases of two null $m$-planes. From (3.1) and the fact that $X_{A}, Y_{1}, Y_{2}$ form a complete system of independent solutions of (3.2), it follows easily by taking the covariant derivative of (3.2) that

$$
\nabla_{k} Y_{1}^{h}=\tilde{L}_{k 1}^{A} X_{A}^{h}+N_{k 1}^{1} Y_{1}^{h}+N_{k 1}^{2} Y_{2}^{h}, \quad \nabla_{k} Y_{2}^{h}=\widetilde{L}_{k 2}^{A} X_{A}^{h}+N_{k 2}^{1} Y_{1}^{h}+N_{k 2}^{2} Y_{2}^{h},
$$

where the $\tilde{L}$ 's and $N$ 's are some covectors. Multiplying these two equations by $Y_{1 h}$ and $Y_{2 h}$, respectively (and summing over $h$ ), we get $N_{k 1}^{2}=0, N_{k 2}^{1}=0$. Therefore,

$$
\nabla_{k} Y_{1}^{h}=\widetilde{L}_{k 1}^{A} X_{A}^{h}+N_{k 1}^{1} Y_{1}^{h}, \quad \nabla_{k} Y_{2}^{h}=\tilde{L}_{k 2}^{A} X_{A}^{h}+N_{k 2}^{2} Y_{2} .
$$


Hence, by (3.1), (3.4) and Proposition (b), the two null $m$-planes with bases $\left\{X_{A}, Y_{1}\right\},\left\{X_{A}, Y_{2}\right\}$ are both parallel $m$-planes on $U$. Obviously these two $m$-planes are contained in the $(m+1)$-plane $\Pi^{\prime}$ with basis $\left\{X_{A}, Y_{1}, Y_{2}\right\}$, which is conjugate to the null-plane $\Pi$ with basis $\left\{X_{A}\right\}$ and is easily seen to be of nullity $m-1$. These prove that Theorem (a) is true in any coordinate neighbourhood $U$ of $M$.

To prove that it is true globally on $M$, let us consider another coordinate neighbourhood $U^{*}$ in $M$ and the vectors $X_{A^{*}}^{*}\left(1 \leqq A^{*} \leqq m-1\right)$, $Y_{1}^{*}$ and $Y_{2}^{*}$ on $U^{*}$ corresponding to the vectors $X_{A}, Y_{1}$ and $Y_{2}$ on $U$. Then, since the null $m$-plane $\Pi$ is given globally on $M$, we have

$$
\text { on } U \cap U^{*}: X_{A^{*}}^{*}=\stackrel{A}{\phi_{A^{*}}} X_{A} \text {, }
$$

for some functions $\phi_{A^{*}}^{A}$ whose matrix $\left(\phi_{A^{*}}^{A}\right)$ is everywhere of full rank in $U \cap U^{*}$. From (3.5), (3.2) and the equations $X_{A}^{*} \cdot W^{*}=0$ which correspond to (3.2), it follows that

$$
Y_{1}^{*}=\psi_{1}^{A} X_{A}+\chi_{11} Y_{1}+\chi_{12} Y_{2}, \quad Y_{2}^{*}=\psi_{2}^{A} X_{A}+\chi_{21} Y_{1}+\chi_{22} Y_{2} .
$$

Therefore, taking into account (3.3) and the corresponding conditions on $Y_{1}^{*}$ and $Y_{2}^{*}$, we have

$$
\begin{aligned}
& 0=Y_{1}^{*} \cdot Y_{1}^{*}=\chi_{11} \chi_{12} Y_{1} \cdot Y_{2}, \quad 0=Y_{2}^{*} \cdot Y_{2}^{*}=\chi_{21} \chi_{22} Y_{1} \cdot Y_{2}, \\
& 0 \neq Y_{1}^{*} \cdot Y_{2}^{*}=\left(\chi_{11} \chi_{22}+\chi_{12} \chi_{21}\right) Y_{1} \cdot Y_{2}, \quad 0 \neq Y_{1} \cdot Y_{2} .
\end{aligned}
$$

These require either that $\chi_{11} \neq 0, \chi_{12}=0, \chi_{21}=0$ and $\chi_{22} \neq 0$, or that $\chi_{11}=0, \chi_{12} \neq 0, \chi_{21} \neq 0$ and $\chi_{22}=0$. Thus, interchanging $Y_{1}^{*}$ and $Y_{2}^{*}$ if necessary, we have

$$
Y_{1}^{*}=\psi_{1}^{A} X_{A}+\chi_{11} Y_{1}, \quad Y_{2}^{*}=\psi_{2}^{A} X_{A}+\chi_{22} Y_{2},
$$

where $\chi_{11} \neq 0, \chi_{22} \neq 0$. Now equations (3.5) and (3.7) show that the $m$ independent vectors $X_{A}$ and $Y_{1}$ which are defined locally in each coordinate neighbourhood of $M$ piece together to form a (null parallel) $m$-plane on $M$. Similarly for the vectors $X_{A}$ and $Y_{2}$. Hence Theorem (a) is completely proved.

4. Proof of Theorem (b). Again we first prove our assertions in a coordinate neighbourhood $U$ of $M$. Let $\Pi$ be a parallel $m$-plane of nullity $m-1$ on $M$ and $\left\{X_{A}, Y\right\}, 1 \leqq A, B \leqq m-1$, a normal basis of $\Pi$ on $U$, where $X_{A}$ are null vectors and $Y$ is a unit vector. Then we have, easily,

$$
\nabla_{k} X_{B}^{h}=L_{k B}^{A} X_{A}^{h}, \quad \nabla_{k} Y^{h}=L_{k}^{A} X_{A}^{h} .
$$

Equations (4.1) $)_{1}$ show that the null part $\Omega$ with basis $\left\{X_{A}\right\}$ is a parallel $(m-1)$-plane. 
Now consider the system of $m$ independent equations:

$$
X_{\Delta} \cdot W=0, \quad Y \cdot W=0 .
$$

It has exactly $m$ independent solutions, and we may take them to be the $m-1$ vectors $X_{B}$ and another vector $Z$. Then $\left\{X_{A}, Z\right\}$ is a basis of the $m$-plane $\Pi^{\prime}$ conjugate to $\Pi$.

It is easily seen from (4.2) that $Z$ cannot be linearly dependent on the $m$ vectors $X_{A}$ and $Y$. Moreover, $Z$ is nonnull; otherwise, the $m$ independent equations

$$
X_{A} \cdot W=0, \quad Z \cdot W=0
$$

would have $m+1$ independent solutions, namely, $X_{B}, Y$ and $Z$, and this is impossible. Therefore, we may take $Z$ to be a unit vector. Let this be done. Then, we easily deduce from (4.1) and (4.2) that

$$
\nabla_{k} Z^{h}=N_{k}^{A} X_{A}^{h}
$$

Now, let $\alpha$ and $\beta$ be any constants, not both zero. Then the vector $\alpha Y+\beta Z$ is linearly independent of the $m-1$ vectors $X_{A}$. Moreover, it follows from $(4.1)_{2}$ and (4.3) that

$$
\nabla_{k}\left(\alpha Y^{h}+\beta Z^{h}\right)=\left(\alpha L_{k}^{A}+\beta N_{k}^{A}\right) X_{A}^{h} .
$$

This, together with $(4.1)_{1}$, shows that the $m$-plane with basis $\left\{X_{A}, \alpha Y+\beta Z\right\}$ is a parallel $m$-plane on $U$, by Proposition (b). If $\alpha Y+\beta Z$ is nonnull, as will be generally the case, the $m$-plane is of nullity $m-1$; but if the ratio $\alpha$ : $\beta$ has either of the two values that make $\alpha Y+\beta Z$ null, then the $m$-plane is wholly null. All the $m$-planes of the pencil $\left\{X_{A}, \alpha Y+\beta Z\right\}$ are contained in the $(m+1)$-plane with basis $\left\{X_{A}, Y, Z\right\}$, which is conjugate to the null $(m-1)$-plane $\Omega$ with basis $\left\{X_{A}\right\}$ and is obviously of nullity $m-1$. Theorem (b) is thus proved for a coordinate neighbourhood $U$ of $M$.

To prove that it is true globally on $M$, we proceed as in the proof of Theorem (a). Thus, on $U \cap U^{*},\left\{X_{A}, Y\right\}$ and $\left\{X_{A}^{*}, Y^{*}\right\}$ are both normal bases of the given $m$-plane of nullity $m-1$, where $X_{A}, X_{A}^{*}$ are null vectors and $Y, Y^{*}$ are unit vectors. Therefore, we have

$$
\text { on } U \cap U^{*}: \quad X_{A^{*}}^{*}=\phi_{A^{*}}^{A} X_{A}, \quad Y^{*}=\phi^{A} X_{A}+Y,
$$

where the matrix $\left(\phi_{A *}^{A}\right)$ is of full rank. On account of this, the equations

$$
X_{A^{*}}^{*} \cdot W^{*}=0, \quad Y^{*} \cdot W^{*}=0,
$$

which correspond to (4.2) reduce to $X_{A} \cdot W^{*}=0, Y \cdot W^{*}=0$. 
Therefore, since both $Z$ and $Z^{*}$ are unit vectors, we have that

$$
Z^{*}=\psi^{A} X_{A}+Z \text {. }
$$

It now follows from (4.5) and (4.6) that, for any constants $\alpha$ and $\beta$, not both zero,

$$
\alpha Y^{*}+\beta Z^{*}=\left(\alpha \phi^{A}+\beta \psi^{A}\right) X_{A}+\alpha Y+\beta Z .
$$

Equations (4.5) 1 and (4.7) show that, for each pair of constants $\alpha$ and $\beta /$ which are not both zero and which are the same for all the coordinate neighbourhoods of $M$, the $m-1$ independent vectors $X_{A}$ and $\alpha Y+\beta Z$ defined locally on each coordinate neighbourhood of $M$ piece together to form a (parallel) $m$-plane of nullity $m-1$ or $m$ on $M$. Hence, Theorem (b) is completely proved.

5. Proof of Theorem (c). The proof is similar to that for Theorem (a) and (b), and only an outline of it will be given. Let $I$ be the given parallel $m$-plane of nullity $m-1$ on $M, U$ a coordinate neighbourhood in $M$ and $\left\{X_{A}, Y\right\}$, where $Y$ is a unit vector, a normal basis of $\Pi$ on $U$. The system of $m$ independent equations $X_{A} \cdot W=0, Y \cdot W=0$ admits exactly $m+1$ independent solutions which may be taken to be the $m-1$ vectors $X_{B}$ and two other vectors $Z_{1}$ and $Z_{2}$ such that

$$
Z_{1} \cdot Z_{1}=0, \quad Z_{2} \cdot Z_{2}=0, \quad Z_{1} \cdot Z_{2} \neq 0 .
$$

Then the two null $m$-planes $\Pi_{1}, \Pi_{2}$ with bases $\left\{X_{A}, Z_{1}\right\},\left\{X_{A}, Z_{2}\right\}$ are both parallel $m$-planes on $U$. The $(m+1)$-planes $\Pi_{1}{ }^{\prime}, \Pi_{2}{ }^{\prime}$ conjugate to $\Pi_{1}, \Pi_{2}$ have bases $\left\{X_{A}, Y, Z_{1}\right\},\left\{X_{A}, Y, Z_{2}\right\}$, respectively. All the planes $\Pi, \Pi_{1}, \Pi_{2}, \Pi_{1}{ }^{\prime}$ and $\Pi_{2}{ }^{\prime}$ in tersect in the null $(m-1)$-plane $\Omega$ with basis $\left\{X_{A}\right\}$ and are contained in the $(m+2)$-plane with basis $\left\{X_{A}, Y, Z_{1}, Z_{2}\right\}$, which is conjugate to $\Omega$.

The proof that these results hold globally on $M$ is almost identical with that given for Theorem (a) and (b) and is therefore omitted.

\section{REFERENCES}

1. H. S. Ruse, On parallel fields of planes in a Riemannian space, Quart. J. Math. Oxford Ser. 20 (1949), 218-234.

2. - Parallel planes in a Riemannian $V_{n}$, Proc. Roy. Soc. Edinburgh Sect. A 68 (1950), 78-92.

3. A. G. Walker, On parallel fields of partially null vector spaces, Quart. J. Math. Oxford Ser. 20 (1949), 135-145.

4. Yung-Chow Wong, Quasi-orthogonal ennuple of congruences in a Riemannian space, Ann. of Math. 46 (1945), 158-173.

5. - Linear connexions with zero torsion and recurrent curvature, Trans. Amer. Math. Soc. 102 (1962), 471-506.

University of HoNg KoNG 